\title{
Y-STR haplotype diversity and population data for Central Brazil: implications for environmental forensics and paternity testing
}

\author{
T.C. Vieira ${ }^{1,2,3,4}$, M.A.D. Gigonzac ${ }^{2,3,4}$, D.M. Silva ${ }^{1}$, R.G. Rodovalho ${ }^{5}$, \\ G.S. Santos ${ }^{5}$ and A.D. da Cruz Cr,2,3 $^{1,2}$ \\ ${ }^{1}$ Programa de Pós-Graduação em Biologia, Instituto de Ciências Biológicas, \\ Universidade Federal de Goiás, Goiânia, GO, Brasil \\ ${ }^{2}$ Laboratório de Citogenética Humana e Genética Molecular, \\ Laboratório Central de Saúde Pública Dr. Giovanni Cysneiros, \\ Secretaria de Estado da Saúde/Governo de Goiás, Goiânia, GO, Brasil \\ ${ }^{3}$ Núcleo de Pesquisas Replicon, Departamento de Biologia, \\ Pontifícia Universidade Católica de Goiás, Goiânia, GO, Brasil \\ ${ }^{4}$ Universidade Estadual de Goiás, Goiânia, GO, Brasil \\ ${ }^{5}$ Laboratório Biocroma, Laboratório de Análise de DNA, Goiânia, GO, Brasil \\ Corresponding author: T.C. Vieira \\ E-mail: thaiscidalia@gmail.com
}

Genet. Mol. Res. 13 (2): 3404-3410 (2014)

Received August 20, 2013

Accepted November 8, 2013

Published April 30, 2014

DOI http://dx.doi.org/10.4238/2014.April.30.1

ABSTRACT. The central region of Brazil was colonized by internal
migration of individuals of different origins, who contributed to the
genetic diversity existing in this population. This study determined
the allele frequencies and haplotype diversity of Y-STRs in Goiás
State, Central Brazil, and compared the data obtained with a sample
of the Brazilian population, consisting of individuals from the five
geographical regions of Brazil. A total of 353 males were typed for 
12 Y-chromosome short tandem repeat (Y-STR) markers. We selected males who had no degree of relatedness, from the five mesoregions of Goiás State. DNA was extracted from blood samples followed by the amplification of the $12 \mathrm{Y}$-chromosome loci. The products were analyzed to obtain the allele profiles on an ABI3500 automated sequencer using the Gene Mapper software. Allele frequencies and haplotype diversity were estimated by direct counting, and gene diversity for each locus was computed using the Arlequin software. The results are consistent with the history of miscegenation of the population of Central Brazil, in which we observed 321 different haplotypes. The average gene diversity at the 12 loci was 0.645 . DYS385b and DYS389I showed the highest $(0.704)$ and lowest $(0.520)$ genetic diversity values, respectively. The $F_{\mathrm{ST}}$ value between the Brazilian and Goiás populations was 0.00951, showing no statistical significance. The results of this study allowed the establishment of haplotypes found in the forensic samples of Goiás State serving as a reference in the elucidation of criminal cases and paternity tests, as well as population and evolutionary inferences.

Key words: Y chromosome; STR markers; Haplotype diversity

\section{INTRODUCTION}

The analysis of Y-chromosome short tandem repeats (STRs) has been extensively used in forensic genetics and for paternity testing. Furthermore, these polymorphisms serve as valuable markers for ancestry studies, and the main population shows remarkable allele frequency distributions (Palha et al., 2007; Carvalho and Pinheiro, 2013; Vieira et al., 2013).

Due to the presence of the largest non-recombining region in the whole human genome, the $\mathrm{Y}$ chromosome is characterized by a unique inheritance pattern and specificity to males (Rębała and Szczerkowska, 2005). The lack of recombination between Y-chromosome specific markers means that these markers are transmitted as haplotypes in the same way as single locus alleles. The lower effective number of Y-chromosomes in a given population also means that Y-haplotypes/haplogroups tend to show a higher proportion of variation between populations than observed for other markers located on autosomes or X chromosomes (Domingues et al., 2007). Thus, Y-chromosome single tandem repeat (Y-STR) analysis can be very useful in paternity tests in which the alleged father is missing or deceased, through analysis of related individuals (Francez et al., 2012).

Investigations of genetic links using database DNA has stood out in recent years (Chung and Fung, 2013; Mardini et al., 2013). Moreover, the study of human race has been of interest since ancient times. The existence of differences between populations of distinct geographical origins must have been a finding of the first long-distance travelers (Silva et al., 2010). Nowadays, considerable research has been conducted to identify the geographical origin of people and their closeness, from several points of view, as well as the degree of admixture in the genetic composition of a population. Therefore, the aim of this study was to determine the allele frequencies and haplotype diversity of Y-STRs in the population of Goias State, Central Brazil, and to compare the results with population data for samples from all Brazil regions (Mardini et al., 2013). 


\section{MATERIAL AND METHODS}

A total of 353 males were typed for the following 12 Y-chromosomal short tandem repeat (Y-STR) markers: DYS389I, DYS389II, DYS391, DYS437, DYS438, DYS439, DYS19, DYS392, DYS393, DYS390, DYS385I, and DYS385II. We selected the samples of the males who had no degree of relatedness, from five mesoregions comprising Goias State (Central, East, North, Northwest, and South regions).

DNA was extracted from blood samples using a commercial kit (GE Healthcare, UK), followed by the amplification of the 12 Y-chromosome loci (Powerplex ${ }^{\circledR}$ System, Promega Corporation) according to manufacturer instructions. The amplification products were analyzed on an ABI3500 automated sequencer (Life Technology, Applied Biosystems) to obtain the allele profiles, which were analyzed using the GeneMapper software 2.1 (Applied Biosystems). Allele frequencies and haplotype diversity were estimated by direct counting, and gene diversity (GD) for each locus was computed using the Arlequin software, version 3.5 (10,000 permutations) (Excoffier and Lischer, 2010).

\section{RESULTS AND DISCUSSION}

The results are consistent with the history of miscegenation for the population of Central Brazil, in which we observed 321 different haplotypes, indicating an individual discrimination power of 0.91 . The average gene diversity at the 12 loci was 0.645 . The DYS385b and DYS389I showed the highest (0.704) and lowest (0.520) genetic diversity values, respectively (Table 1$)$.

Midwestern Brazil, like other Brazilian populations, is derived from the admixture of 3 main groups: Amerindians, Europeans, particularly Portuguese, and Africans from subSaharan Africa (Vieira et al., 2013). A comparative analysis of our population with published data from other Brazilian populations was performed using the Arlequin software 3.5 (Pereira et al., 2007; Excoffier and Lischer, 2010). Three rare alleles were detected in the Goias population in markers DYS391, DYS392 and DYS438, which were not described in the study of the Brazilian population.

To test the level of genetic differentiation between the population from Goias State and the Brazilian population $\left(F_{\mathrm{ST}}\right)$, intrapopulation genetic diversity $\left(H_{\mathrm{S}}\right)$ and the proportion of the total genetic diversity $\left(H_{\mathrm{T}}\right)$ were estimated using the FSTAT software, version 2.9 (Goudet, 2001) (Table 2). The overall $\phi_{\mathrm{ST}}$ and $R_{\mathrm{ST}}$ values determined by AMOVA were respectively 0.009 and 0.0084 , confirming no significant differentiation for the Y-STR haplotype between the Goiás and Brazilian populations. Allele frequencies and resulting statistical parameters are given in Tables 1 and 2 .

The genetic differentiation coefficient $\left(F_{\mathrm{ST}}\right)$ between the Brazilian and Goias populations was 0.00951 , showing no statistical significance $(\mathrm{P}>0.05)$. An $F_{\mathrm{ST}}$ value between 0 and 0.05 indicates a low level of genetic differentiation, but it does not imply the absence of differentiation.

Forensic genetics interest has focused on Y-STR markers, which are available for population genetic, evolutionary, genealogical, and forensic investigations (Soares-Vieira et al., 2008). The results reported here characterized the genetic diversity of the population of Goias based on Y-STR and contributed to consolidate a database that could be useful in human identification studies. Additionally, the data will support the demands from the Judiciary and Public Ministry in the area of family and criminal affairs, helping in the elucidation of criminal cases and paternity testing for the Goias population, especially when the DNA of the alleged father is not available. 
Table 1. Allele frequencies and genetic diversity values of 12 Y-STRs in 353 men from the Goias population in Central Brazil.

\begin{tabular}{|c|c|c|c|c|c|c|c|c|c|c|c|c|}
\hline Allele & DYS389I & DYS389II & DYS391 & DYS437 & DYS438 & DYS439 & DYS19 & DYS392 & DYS393 & DYS390 & DYS385a & DYS385b \\
\hline 7 & - & - & $0.0028 *$ & - & - & 0.0000 & & - & - & - & - & - \\
\hline 8 & - & - & 0.0000 & - & 0.0057 & 0.0000 & - & - & - & - & - & - \\
\hline 9 & - & - & 0.0682 & - & 0.1178 & 0.0057 & - & 0.0000 & - & - & 0.0028 & - \\
\hline 10 & - & - & 0.4773 & - & 0.2529 & 0.1026 & - & 0.0029 & 0.0031 & - & 0.0028 & - \\
\hline 11 & - & - & 0.4375 & - & 0.1351 & 0.3305 & - & 0.4029 & 0.0951 & - & 0.4986 & 0.0170 \\
\hline 12 & 0.1648 & - & 0.0142 & 0.0000 & 0.4511 & 0.4074 & 0.0085 & 0.0600 & 0.4294 & - & 0.0997 & 0.0398 \\
\hline 13 & 0.6477 & - & - & 0.0085 & 0.0345 & 0.1481 & 0.1225 & 0.4714 & 0.3865 & - & 0.1681 & 0.0284 \\
\hline 14 & 0.1875 & - & - & 0.3371 & $0.0029 *$ & 0.0057 & 0.5499 & 0.0543 & 0.0736 & - & 0.0969 & 0.4943 \\
\hline 15 & 0.0000 & - & - & 0.5411 & - & - & 0.2137 & 0.0057 & 0.0123 & - & 0.0427 & 0.1903 \\
\hline 16 & 0.0000 & - & - & 0.1105 & - & - & 0.0798 & $0.0029 *$ & - & - & 0.0598 & 0.0795 \\
\hline 17 & - & - & - & 0.0028 & - & - & 0.0256 & - & - & - & 0.0228 & 0.0653 \\
\hline 18 & - & - & - & - & - & - & - & - & - & - & 0.0057 & 0.0568 \\
\hline 19 & - & - & - & - & - & - & - & - & - & - & 0.0000 & 0.0227 \\
\hline 20 & - & - & - & - & - & - & - & - & - & 0.0000 & - & 0.0057 \\
\hline 21 & - & - & - & - & - & - & - & - & - & 0.0595 & - & 0.00000 \\
\hline 22 & - & - & - & - & - & - & - & - & - & 0.0708 & - & - \\
\hline 23 & - & - & - & - & - & - & - & - & - & 0.2578 & - & - \\
\hline 24 & - & - & - & - & - & - & - & - & - & 0.4929 & - & - \\
\hline 25 & - & - & - & - & - & - & - & - & - & 0.1105 & - & - \\
\hline 26 & - & 0.0057 & - & - & - & - & - & - & - & 0.0028 & - & - \\
\hline 27 & - & 0.0028 & - & - & - & - & - & - & - & 0.0028 & - & - \\
\hline 28 & - & 0.1080 & - & - & - & - & - & - & - & - & - & - \\
\hline 29 & - & 0.4261 & - & - & - & - & - & - & - & - & - & - \\
\hline 30 & - & 0.3125 & - & - & - & - & - & - & - & - & - & - \\
\hline 31 & - & 0.1136 & - & - & - & - & - & - & - & - & - & - \\
\hline 32 & - & 0.0284 & - & - & - & - & - & - & - & - & - & - \\
\hline 33 & - & 0.0028 & - & - & - & - & - & - & - & - & - & - \\
\hline 34 & - & - & - & - & - & - & - & - & - & - & - & - \\
\hline $\mathrm{N}$ & 352 & 352 & 352 & 353 & 348 & 351 & 351 & 350 & 331 & 353 & 351 & 352 \\
\hline GD & 0.520 & 0.697 & 0.578 & 0.583 & 0.701 & 0.694 & 0.632 & 0.611 & 0.657 & 0.672 & 0.700 & 0.704 \\
\hline
\end{tabular}

$\mathrm{N}=$ number of individuals; GD = gene diversity; *rare alleles.

Table 2. Allele frequencies of Brazilian and Goiás populations, intrapopulation genetic diversity for each locus $\left(H_{\mathrm{S}}\right)$, total genetic diversity for each locus $\left(H_{\mathrm{T}}\right)$ and genetic differentiation coefficient between populations $\left(F_{\mathrm{ST}}\right)$.

\begin{tabular}{lcc}
\hline Locus & Goiás population & Brazilian population \\
\hline DYS439 & 351 & 480 \\
$(\mathrm{~N})$ & 0.0000 & 0.0021 \\
7 & 0.0000 & 0.0021 \\
8 & 0.0057 & 0.0062 \\
9 & 0.1026 & 0.0561 \\
10 & 0.3305 & 0.3929 \\
11 & 0.4074 & 0.3992 \\
12 & 0.1481 & 0.1227 \\
13 & 0.0057 & 0.0187 \\
14 & $H_{\mathrm{T}}=0.6817$ & $F_{\mathrm{ST}}$ \\
$H_{\mathrm{S}}=0.6800$ & $H_{\mathrm{T}}(\mathrm{nc})=0.6825$ & 0.00264 \\
$H_{\mathrm{S}}(\mathrm{nc})=0.6817$ & & 480 \\
$\mathrm{DYS} 19$ & 351 & 0.0042 \\
$(\mathrm{~N})$ & 0.0085 & 0.1333 \\
12 & 0.1225 & 0.5188 \\
13 & 0.5499 & 0.2542 \\
14 & 0.2137 & 0.0583 \\
15 & 0.0798 & 0.0313 \\
16 & 0.0256 & $F_{\mathrm{ST}}$ \\
17 & $H_{\mathrm{T}}=0.6378$ & 0.00006 \\
$H_{\mathrm{S}}=0.6370$ & $H_{\mathrm{T}}(\mathrm{nc})=0.6386$ & \\
$H_{\mathrm{S}}(\mathrm{nc})=0.6486$ & & \\
\hline & & \\
\hline
\end{tabular}

Continued on next page 
T.C. Vieira et al.

3408

\section{Table 2. Continued.}

\begin{tabular}{|c|c|c|}
\hline Locus & Goiás population & Brazilian population \\
\hline \multicolumn{3}{|l|}{ DYS389I } \\
\hline$(\mathrm{N})$ & 352 & 481 \\
\hline 10 & 0.0000 & 0.0021 \\
\hline 11 & 0.0000 & 0.0104 \\
\hline 12 & 0.1648 & 0.1705 \\
\hline 13 & 0.6477 & 0.6466 \\
\hline 14 & 0.1875 & 0.1622 \\
\hline 15 & 0.0000 & 0.0083 \\
\hline$H_{\mathrm{S}}=0.5223$ & $H_{\mathrm{T}}=0.5225$ & $F_{\mathrm{ST}}=-0.00164$ \\
\hline$H_{\mathrm{S}}(\mathrm{nc})=0.5236$ & $H_{\mathrm{T}}(\mathrm{nc})=0.5231$ & \\
\hline \multicolumn{3}{|l|}{ DYS389II } \\
\hline (N) & 352 & 481 \\
\hline 26 & 0.0057 & 0.0042 \\
\hline 27 & 0.0028 & 0.0125 \\
\hline 28 & 0.1080 & 0.1351 \\
\hline 29 & 0.4261 & 0.4699 \\
\hline 30 & 0.3125 & 0.2640 \\
\hline 31 & 0.1136 & 0.0894 \\
\hline 32 & 0.0284 & 0.0229 \\
\hline 33 & 0.0028 & 0.0021 \\
\hline$H_{\mathrm{S}}=0.6889$ & $H_{\mathrm{T}}=0.6904$ & $F_{\mathrm{ST}}=0.00168$ \\
\hline$H_{\mathrm{S}}(\mathrm{nc})=0.6906$ & $H_{\mathrm{T}}(\mathrm{nc})=0.6912$ & \\
\hline \multicolumn{3}{|l|}{ DYS390 } \\
\hline$(\mathrm{N})$ & 353 & 481 \\
\hline 20 & 0.0000 & 0.0021 \\
\hline 21 & 0.0595 & 0.0437 \\
\hline 22 & 0.0708 & 0.1019 \\
\hline 23 & 0.2578 & 0.2911 \\
\hline 24 & 0.4929 & 0.4699 \\
\hline 25 & 0.1105 & 0.0852 \\
\hline 26 & 0.0028 & 0.0062 \\
\hline 27 & 0.0028 & 0.0000 \\
\hline$H_{\mathrm{S}}=0.6724$ & $H_{\mathrm{T}}=0.6732$ & $F_{\mathrm{ST}}=0.00016$ \\
\hline$H_{\mathrm{S}}(\mathrm{nc})=0.6740$ & $H_{\mathrm{T}}(\mathrm{nc})=0.6741$ & \\
\hline \multicolumn{3}{|l|}{ DYS391 } \\
\hline$(\mathrm{N})$ & 352 & 480 \\
\hline 7 & 0.0028 & 0.0000 \\
\hline 8 & 0.0000 & 0.0021 \\
\hline 9 & 0.0682 & 0.0542 \\
\hline 10 & 0.4773 & 0.5146 \\
\hline 11 & 0.4375 & 0.4146 \\
\hline 12 & 0.0142 & 0.0146 \\
\hline$H_{\mathrm{S}}=0.5681$ & $H_{\mathrm{T}}=0.5686$ & $F_{\mathrm{ST}}=-0.00059$ \\
\hline$H_{\mathrm{S}}(\mathrm{nc})=0.5695$ & $H_{\mathrm{T}}(\mathrm{nc})=0.5693$ & \\
\hline \multicolumn{3}{|l|}{ DYS392 } \\
\hline$(\mathrm{N})$ & 350 & 481 \\
\hline 9 & 0.0000 & 0.0021 \\
\hline 10 & 0.0029 & 0.0021 \\
\hline 11 & 0.4029 & 0.4179 \\
\hline 12 & 0.0600 & 0.0499 \\
\hline 13 & 0.4714 & 0.4636 \\
\hline 14 & 0.0543 & 0.0561 \\
\hline 15 & 0.0057 & 0.0083 \\
\hline 16 & 0.0029 & 0.0000 \\
\hline$H_{\mathrm{S}}=0.6068$ & $H_{\mathrm{T}}=0.6069$ & $F_{\mathrm{ST}}=-0.00213$ \\
\hline$H_{\mathrm{S}}(\mathrm{nc})=0.6083$ & $H_{\mathrm{T}}(\mathrm{nc})=0.6076$ & \\
\hline
\end{tabular}

Continued on next page 
Table 2. Continued.

\begin{tabular}{|c|c|c|}
\hline Locus & Goiás population & Brazilian population \\
\hline \multicolumn{3}{|l|}{ DYS393 } \\
\hline$(\mathrm{N})$ & 331 & 481 \\
\hline 10 & 0.0000 & 0.0021 \\
\hline 11 & 0.0906 & 0.0083 \\
\hline 12 & 0.4139 & 0.1726 \\
\hline 13 & 0.3988 & 0.6819 \\
\hline 14 & 0.0816 & 0.1102 \\
\hline 15 & 0.0151 & 0.0249 \\
\hline$H_{\mathrm{S}}=0.5735$ & $H_{\mathrm{T}}=0.6100$ & $F_{\mathrm{ST}}=0.11342$ \\
\hline$H_{\mathrm{S}}^{\mathrm{s}}(\mathrm{nc})=0.5749$ & $H_{\mathrm{T}}(\mathrm{nc})=0.6107$ & \\
\hline \multicolumn{3}{|l|}{ DYS385a } \\
\hline$(\mathrm{N})$ & 351 & 480 \\
\hline 9 & 0.0028 & 0.0083 \\
\hline 10 & 0.0028 & 0.0146 \\
\hline 11 & 0.4986 & 0.4437 \\
\hline 12 & 0.0997 & 0.0979 \\
\hline 13 & 0.1681 & 0.1937 \\
\hline 14 & 0.0969 & 0.0917 \\
\hline 15 & 0.0427 & 0.0458 \\
\hline 16 & 0.0598 & 0.0646 \\
\hline 17 & 0.0228 & 0.0313 \\
\hline 18 & 0.0057 & 0.0063 \\
\hline 19 & 0.0000 & 0.0021 \\
\hline$H_{\mathrm{S}}=0.7189$ & $H_{\mathrm{T}}=0.7199$ & $F_{\mathrm{ST}}=0.00028$ \\
\hline$H_{\mathrm{S}}(\mathrm{nc})=0.7207$ & $H_{\mathrm{T}}(\mathrm{nc})=0.7208$ & \\
\hline \multicolumn{3}{|l|}{ DYS385b } \\
\hline$(\mathrm{N})$ & 353 & 481 \\
\hline 11 & 0.0170 & 0.0083 \\
\hline 12 & 0.0398 & 0.0208 \\
\hline 13 & 0.0284 & 0.0644 \\
\hline 14 & 0.4943 & 0.4532 \\
\hline 15 & 0.1903 & 0.1642 \\
\hline 16 & 0.0795 & 0.0811 \\
\hline 17 & 0.0653 & 0.1040 \\
\hline 18 & 0.0568 & 0.0603 \\
\hline 19 & 0.0227 & 0.0291 \\
\hline 20 & 0.0057 & 0.0125 \\
\hline 21 & 0.0000 & 0.0021 \\
\hline$H_{\mathrm{S}}=0.7217$ & $H_{\mathrm{T}}=0.7231$ & $F_{\mathrm{ST}}=0.00146$ \\
\hline$H_{\mathrm{S}}(\mathrm{nc})=0.7234$ & $H_{\mathrm{T}}(\mathrm{nc})=0.7240$ & \\
\hline \multicolumn{3}{|l|}{ DYS437 } \\
\hline$(\mathrm{N})$ & 353 & 481 \\
\hline 12 & 0.0000 & 0.0021 \\
\hline 13 & 0.0085 & 0.0042 \\
\hline 14 & 0.3371 & 0.3555 \\
\hline 15 & 0.5411 & 0.5177 \\
\hline 16 & 0.1105 & 0.1123 \\
\hline 17 & 0.0028 & 0.0083 \\
\hline$H_{\mathrm{S}}=0.5871$ & $H_{\mathrm{T}}=0.5874$ & $F_{\mathrm{ST}}=-0.00166$ \\
\hline$H_{\mathrm{S}}^{3}(\mathrm{nc})=0.5886$ & $H_{\mathrm{T}}(\mathrm{nc})=0.5881$ & \\
\hline \multicolumn{3}{|l|}{ DYS438 } \\
\hline (N) & 348 & 481 \\
\hline 8 & 0.0057 & 0.0042 \\
\hline 9 & 0.1178 & 0.1351 \\
\hline 10 & 0.2529 & 0.2973 \\
\hline 11 & 0.1351 & 0.0811 \\
\hline 12 & 0.4511 & 0.4636 \\
\hline 13 & 0.0345 & 0.0187 \\
\hline 14 & 0.0029 & 0.0000 \\
\hline$H_{\mathrm{s}}=0.6853$ & $H_{\mathrm{T}}=0.06867$ & $F_{\mathrm{ST}}=0.00161$ \\
\hline$H_{\mathrm{S}}^{\mathrm{s}}(\mathrm{nc})=0.6870$ & $H_{\mathrm{T}}(\mathrm{nc})=0.6876$ & \\
\hline
\end{tabular}

$H_{\mathrm{S}}=$ intrapopulation genetic diversity for each locus; $H_{\mathrm{S}}(\mathrm{nc})=$ intrapopulation genetic diversity not corrected for each locus; $H_{\mathrm{T}}=$ total genetic diversity for each locus; $H_{\mathrm{T}}(\mathrm{nc})=$ total genetic diversity not corrected for each locus; $F_{\mathrm{ST}}=$ genetic differentiation coefficient between populations. 


\section{ACKNOWLEDGMENTS}

Research supported by FAPEG-GO (Fundação de Amparo à Pesquisa do Estado de Goiás) and CNPq (for T.C. Vieira scholarship). We thank the participants who voluntarily donated blood samples for this study. We are also grateful to the staff from Núcleo de Pesquisas Replicon da Pontifícia Universidade Católica de Goiás and Laboratório de Citogenética Humana- Genética Molecular/Secretaria de Estado da Saúde (LaGene/Lacen/SES-GO). We also thank COAM and S. Quail for English support.

\section{REFERENCES}

Carvalho A and Pinheiro MF (2013). Population data of 30 insertion/delection polymorphisms from a sample taken in the North of Portugal. Int. J. Legal Med. 127: 65-67.

Chung YK and Fung WK (2013). Identifying contributors of two-person DNA mixtures by familial database search. Int. J. Legal Med. 127: 25-33.

Domingues PM, Gusmão L, da Silva DA, Amorim A, et al. (2007). Sub-Saharan Africa descendents in Rio de Janeiro (Brazil): population and mutational data for 12 Y-STR loci. Int. J. Legal Med. 121: 238-241.

Excoffier L and Lischer HE (2010). Arlequin suite ver 3.5: a new series of programs to perform population genetics analyses under Linux and Windows. Mol. Ecol. Resour. 10: 564-567.

Francez PA, Ramos LP, de Jesus Brabo Ferreira Palha and Dos Santos SE (2012). Haplotype diversity of 17 Y-str loci in an admixed population from the Brazilian Amazon. Genet. Mol. Biol. 35: 45-52.

Goudet J (2001). FSTAT, A Program to Estimate and Test Gene Diversities and Fixation Indices (Ver. 2·9·3). Available at [http://www2.unil.ch/popgen/softwares/fstat.htm]. Accessed November 5, 2013.

Mardini AC, Rodenbusch R, Schumacher S, Chula FG, et al. (2013). Mutation rate estimates for 13 STR loci in a large population from Rio Grande do Sul, Southern Brazil. Int. J. Legal Med. 127: 45-47.

Palha TJBF, Rodrigues EMR and Santos SEB (2007). Y-chromossomal STR haplotypes in population from the Amazon region, Brazil. Forensic Sci. Int. 166: 233-239.

Pereira RW, Monteiro EH, Hirschfeld GC, Wang AY, et al. (2007). Haplotype diversity of 17 Y-chromosome STRs in Brazilians. Forensic Sci. Int. 171: 226-236.

Rębała K and Szczerkowska Z (2005). Polish population study on Y chromosome haplotypes defined by 18 STR loci. Int. J. Legal Med. 119: 303-305.

Silva MB, Silva DM, Rodovalho RG, Rios PA, et al. (2010). Allele frequencies of fifteen STR loci in a population from Central Brazil. Forensic Sci. Int. Genet. 4: e151-e152.

Soares-Vieira JA, Billerbeck AEC, Iwamura ESM and Mendonca BB (2008). Population and Mutation analysis of Y-STR loci in a sample from the city of São Paulo (Brazil). Genet. Mol. Biol. 31: 651-656.

Vieira TC, Silva DM, Gigonzac MA, Ferreira VL, et al. (2013). Allelic frequencies and statistical data obtained from 15 STR loci in a population of the Goias State. Genet. Mol. Res. 12: 23-27. 